\title{
Monitorización Ambulatoria de Presión Arterial (MAPA): recomendaciones de la rama de nefrología pediátrica
}

\author{
Ambulatory Blood Pressure Monitoring (ABPM): statement \\ from chilean pediatric nephrology committee
}

\section{Daniela Carrillo ${ }^{\mathrm{a}, \mathrm{b}}$, Claudia González ${ }^{\mathrm{a}, \mathrm{b}}$, María Luisa Ceballos ${ }^{\mathrm{c}}$, Paulina Salas ${ }^{\mathrm{d}}$, Lillian Bolte ${ }^{\mathrm{e}, \mathrm{f}}$, Claudia Alarcón ${ }^{\mathrm{g}}$, Soledad Peredo ${ }^{\mathrm{b}}$}

\author{
aHospital Sótero del Río \\ bPontificia Universidad Católica de Chile \\ cHospital Luis Calvo Mackenna \\ dHospital Exequiel González Cortés \\ eHospital Roberto del Río \\ ${ }^{\dagger}$ Clínica Alemana \\ ${ }^{9 H o s p i t a l ~ F e ́ l i x ~ B u l n e s ~}$
}

\begin{abstract}
Resumen
La monitorización ambulatoria de la presión arterial (MAPA) es una herramienta clínica útil para el diagnóstico y confirmación de hipertensión arterial en pediatría y permite igualmente el diagnóstico de condiciones especiales como la hipertensión de delantal blanco e hipertensión enmascarada. Existen recomendaciones internacionales para su realización e interpretación, sin embargo, aún quedan interrogantes por resolver. En esta guía se resume la bibliografía disponible y se intenta estandarizar, a través de consenso de especialistas nacionales, la aplicación de esta técnica. Se necesitan más estudios de investigación en niños que aporten nuevos valores de referencia y que determinen la relación de alteraciones en MAPA con resultados clínicos a largo plazo.
\end{abstract}

Palabras clave:

HTA;

MAPA;

daño órgano blanco;

Dip nocturno

Correspondencia:

Daniela Carrillo

danielacarrillov@gmail.com 


\begin{abstract}
Ambulatory blood pressure monitoring (ABPM) is a useful clinical tool for the diagnosis and confirmation of arterial hypertension in pediatrics, and also allows the diagnosis of special conditions such as white coat hypertension and masked hypertension. There are international recommendations for its implementation and interpretation, however, there are still unresolved questions. This guide summarizes the available literature and attempts to standardize, through consensus of national specialists, the application of this technique. More research studies are needed that provide new reference values and determine the relationship of alterations in ABPM with long-term clinical results.
\end{abstract}

Keywords:

HBP;

ABPM;

target organ damage;

Dipping

\section{Introducción}

La prevalencia de hipertensión arterial (HTA) en pediatría ha ido en sostenido aumento en el último tiempo, asociado al incremento de las cifras de obesidad, siendo un predictor de desarrollo de HTA y enfermedad cardiovascular en la adultez.

La monitorización ambulatoria de la presión arterial (MAPA) es una herramienta clínica útil que proporciona una descripción más precisa de la presión arterial (PA) en relación a las obtenidas en la consulta médica. En población adulta se recomienda realizar un MAPA para confirmar la HTA antes de iniciar tratamiento antihipertensivo ${ }^{1}$.

En pediatría, MAPA es un examen que aún no está disponible en forma universal y existen interrogantes por resolver que permitan una óptima interpretación en niños. Sin embargo, se ha demostrado que MAPA sería más preciso en el diagnóstico de HTA que las presiones arteriales clínicas tomada en el control médico $^{2,3}$, además sería útil en la evaluación de HTA secundaria ${ }^{4}$ y en múltiples patologías con riesgo cardiovascular aumentado.

El uso rutinario de MAPA permite evaluar la severidad y caracterización de la HTA, lo que finalmente estratifica el riesgo cardiovascular. Es importante destacar que un MAPA confirmatorio de HTA tiene mayor correlación con daño de órgano blanco, especialmente hipertrofia ventricular izquierda (HVI), que la HTA diagnosticada en la consulta médica ${ }^{5,6}$.

Además, evalúa la presencia de HTA nocturna, la cual se asocia a mayor incidencia de morbilidad cardiovascular, HVI y progresión de enfermedad renal crónica $(\mathrm{ERC})^{7-9}$.

En pacientes con ERC, MAPA ha demostrado ser más preciso en el diagnóstico de HTA en comparación con la PA clínica ${ }^{8}$, describiéndose una prevalencia de HTA enmascarada en este grupo de alrededor del $38 \%{ }^{9}$, la cual sólo puede ser diagnosticada a través de este método. Existen estudios que han demostrado que pacientes con ERC e HTA en tratamiento presentan un $23 \%$ de inadecuado control de la PA clínica, el cual aumenta a $47 \%$ cuando se realiza MAPA, motivo por el cual la ERC es una de las patologías en las cuales este examen se debe realizar durante su seguimiento ${ }^{10}$.

Existen recomendaciones pediátricas internacionales de la American Heart Association (AHA) específicas para $\mathrm{MAPA}^{7,11}$. Sin embargo, aún quedan interrogantes por resolver tanto en los aspectos técnicos como en la interpretación de las variables que están incluidas en este examen. Este artículo tiene como objetivo dar a conocer las recomendaciones para la interpretación de MAPA sugeridas internacionalmente y generar un consenso, a nivel de la rama de Nefrología Infantil, de aquellos puntos que aún son controversiales en la interpretación de este examen.

\section{Indicaciones y beneficios de MAPA}

De acuerdo con las recomendaciones de la Academia Americana de Pediatría (AAP), el MAPA debiera ser solicitado a niños y adolescentes que presenten PA elevada por un año o más y en pacientes con HTA estadio I en tres controles médicos sucesivos ${ }^{12}$. Además, debe realizarse de rutina en niños y adolescentes con patologías con alto riesgo de HTA para evaluar su presencia y severidad (tabla 1 ).

El MAPA permite:

- Describir y caracterizar diferentes patrones de HTA.

- Describir el patrón circadiano de la PA.

- Evaluar la presencia de HTA del delantal blanco.

- Evaluar la presencia de HTA enmascarada.

- Evaluar PA en pacientes con alto riesgo de daño de órgano blanco.

- Evaluar la eficacia de la terapia hipotensora.

- Diagnosticar hiper/hipotensión relacionada a uso de medicamentos.

\section{Realización de MAPA: Aspectos técnicos}

Se recomienda realizar el examen a partir de los 5 años.

\section{Entrevista:}

- Evaluar contraindicaciones para MAPA (trastornos de coagulación, arritmias, alergia al látex). 
Tabla 1 Condiciones clínicas en las cuales se recomienda realizar MAPA

- Confirmación diagnóstica de HTA

- HTA secundaria

- HTA en tratamiento

- ERC o malformaciones renales

- Diabetes Mellitus (DM) 1 y 2

- Trasplante de órganos sólidos y Trasplante de médula ósea

- Obesidad

- Síndrome apnea obstructiva del sueño (SAOS)

- Coartación aórtica operada

- Síndromes genéticos asociados con HTA (neurofibromatosis, Sd Turner, Sd Williams)

- Prematuros (<32 sem)

- PEG severo

- Protocolos de investigación

- Constatar antecedentes familiares y personales asociados a HTA

- Registrar medicamentos que esté recibiendo con sus respectivos horarios.

- Indicar al paciente que registre en un diario las actividades relevantes durante el día: hora de despertar, de dormirse, ejercicio y jugar video juegos, dosis y horarios de medicamentos, situaciones de stress o dolor, síntomas de hiper/hipotensión.

\section{Instalación manguito}

- Utilizar equipos validados por Association for the Advancement of Medical Instrumentation US national standard (AAM) or the British Hypertension Society standard (BHS). (www.dableducational. org; www.bhsoc.org). Considerar disponibilidad de servicio técnico local.

- Idealmente realizar calibración del equipo en forma anual.
- Instalar el manguito en brazo no dominante (excepto cirugías arteriales, fístulas). Usar manguito acorde a recomendaciones ${ }^{7,12}$.

III. Tomar PA con método oscilométrico tradicional y luego con equipo de MAPA por 3 veces y promediar los dos últimos valores de PA obtenidas por cada método. La diferencia entre ambos equipos debe ser menor a $5 \mathrm{mmHg}$. Si es mayor, reajustar el manguito o chequear calibración del equipo ${ }^{12}$.

\section{IV.Programación de quipo}

- Programar equipo según tabla 2.

\section{Registrar valores de referencia de PA para realizar MAPA:}

- A diferencia de los adultos que poseen una única cifra límite tanto diurna como nocturna que les permite diagnosticar hipertensión, en niños existen diferentes valores dependiendo del género, la edad y la talla, las que se recomiendan mantener hasta los 18 años.

- Se deben utilizar los valores de referencia de las tablas de Wühl et al. ${ }^{7}$, basadas en sexo y talla para niños que midan más de $120 \mathrm{~cm}$ y basadas en edad para niños $<120 \mathrm{~cm}$ de longitud. Se programará el equipo con el valor equivalente a p95 de PA para el día y noche respectivamente.

- Las tablas según talla están distribuidas cada $5 \mathrm{~cm}$ de estatura, por lo que al elegir el valor de corte para determinar el p95, se recomienda aproximar la talla al valor más cercano; para esto se dividirán los $5 \mathrm{~cm}$ por la mitad, donde a la mitad menor le corresponderá el valor de PA más bajo y a la mitad mayor, el valor de PA más alto. Ejemplo: Entre 120 $\mathrm{cm}$ y $125 \mathrm{~cm}$ : si mide $<122,5 \mathrm{~cm}$, elegir la PA correspondiente a $120 \mathrm{~cm}$ y si mide $\geq 122,5 \mathrm{~cm}$, elegir la PA acorde a $125 \mathrm{~cm}$.

Tabla 2. Parámetros para realizar programación de equipo

\begin{tabular}{lll}
\hline Parámetro & Valor & Comentario \\
\hline Frecuencia registros: & cada $15-20$ minutos & Lecturas válidas: \\
$-\quad$ Diurnos & cada $20-30$ minutos & - mínimo 40 lecturas \\
- Nocturnos & $<220 \mathrm{~mm} \mathrm{Hg}$ & Medimo una por hora \\
Calibración PA sistólica & $>60 \mathrm{~mm} \mathrm{Hg}$ & \\
& $<120 \mathrm{~mm} \mathrm{Hg}$ & Medida fuera de este rango, no válida. \\
Calibración PA diastólica & $>35 \mathrm{~mm} \mathrm{Hg}$ & \\
Calibración frecuencia cardíaca & $40-180 \mathrm{lpm}$ & Medida fuera de este rango, no válida. \\
Calibración presión pulso & $20-120 \mathrm{~mm} \mathrm{Hg}$ * & Medida fuera de este rango, no válida. \\
\end{tabular}


- Las tablas de percentiles están confeccionadas con decimales, por lo que para elegir el valor de PA, se sugiere escoger el valor absoluto de PA menor, sin aproximar hacia arriba.

\section{En caso de fallas en el registro, evaluar:}

- Desconexión del equipo.

- Suspensión de lecturas por apretar botón de "cancelar".

- Apagado de monitor.

- Uso de pilas inadecuadas.

- Movimientos que provoquen artefactos en el registro.

- Alteraciones en las líneas de conexión.

- Revisar equipo y manguitos.

\section{Interpretación de MAPA}

I. Se recomienda considerar estudio técnicamente satisfactorio cuando presente:

- Al menos 40 lecturas totales válidas para un reporte de 24 horas.

- $65 \%$ a $75 \%$ de lecturas válidas para un reporte parcial de día.

- Una lectura por hora durante las 24 horas de examen.

- Se considerará estudio no interpretable con $<$ de 40 lecturas válidas (condición por sí sola) o pérdida de horas de registro ( $\geq 2$ horas consecutivas o 3 horas en distintos momentos del examen).

\section{Interpretación de variables:}

- Describir si el estudio fue interpretable o no.

- La interpretación del MAPA se basa en la evaluación del promedio de las PA y el porcentaje de sobrecarga por sobre p95 para los períodos de vigilia y sueño.
Se considerará alterada: el promedio de $\mathrm{PA} \geq \mathrm{p} 95$ $y$ una sobrecarga de $P A \geq 25 \%$ de lecturas sobre el p95.

- Evaluar la caída fisiológica de PA durante el sueño: calcular DIP nocturno de PAS y PAD (PA promedio diurna-PA promedio nocturna/PA promedio diurna; multiplicado por 100). Se considera DIP nocturno normal, si la disminución de PAS y PAD es mayor al $10 \%$ y menor a $20 \%$.

- Evaluar la PA clínica concomitante: se considerará para la interpretación del examen el promedio de las últimas dos de las tres mediciones, clasificando al paciente según las recomendaciones actuales de la $\mathrm{AAP}^{12}$.

De acuerdo los resultados anteriores, se interpretará el estudio en base a tabla 3 .

\section{Clasificación diagnóstica según MAPA y condiciones clínicas asociadas}

\section{HTA delantal blanco}

Definida como una PA clínica mayor al p95 para sexo, edad y talla con MAPA normal. La prevalencia en pediatría descrita es entre $23-47 \%{ }^{13}$. MAPA tiene un importante rol en la evaluación inicial para evitar estudio y tratamiento innecesario. En cuanto a su significación clínica, en adultos se ha asociado a mayor riesgo cardiovascular por presentar alteración endotelial que favorecería la arterioesclerosis y un mayor riesgo de progresar a HTA persistente. Existen pocos estudios en pediatría que sigan a largo plazo a estos pacientes para ver su riesgo cardiovascular o la posibilidad de desarrollar hipertensión arterial, pero hay 2 estudios recientes ${ }^{13,14}$ que han demostrado alteración en la velocidad de la onda de pulso (rigidez de aorta) y disfunción endotelial en estos niños. Ambos estudios demuestran que estas alteraciones preceden e incluso

Tabla 3. Esquema de clasificación diagnóstica para niños sometidos a MAPA

\begin{tabular}{|c|c|c|c|}
\hline Clasificación & PA en consulta* & Promedio PAS o PAD ambulatoria** & Carga de PAS o PAD en \%** \\
\hline PA normal & $<\mathrm{p} 90$ & $<\mathrm{p} 95$ & $<25$ \\
\hline Hipertensión delantal blanco & $\geq \mathrm{p} 95$ & $<\mathrm{p} 95$ & $<25$ \\
\hline Pre hipertensión & zp90 ó > 120/80 & $<\mathrm{p} 95$ & $\geq 25$ \\
\hline HTA enmascarada & $<\mathrm{p} 95$ & $\geq$ p95 & $\geq 25$ \\
\hline HTA ambulatoria & $\geq$ p95 & $\geq p 95$ & $25-50$ \\
\hline $\begin{array}{l}\text { HTA ambulatoria Severa (con riesgo } \\
\text { de daño órgano blanco) }\end{array}$ & $\geq p 95$ & $\geq p 95$ & $>50$ \\
\hline
\end{tabular}

*Basados en Pediatrics 2017; 140(3): e20171904. **Valores publicados en Hypertension 2008; 52:433-451, tanto para el día como para la noche, o ambos 
hacen sospechar la aparición de arterioesclerosis en forma temprana, tanto en niños con HTA del delantal blanco o esencial. Con estos hallazgos, no existe aún consenso si se debiera o no tratar a estos pacientes, tanto niños como adultos, dado que por estos hallazgos sería una condición no benigna ${ }^{15}$. La AAP recomienda realizar cambios de hábitos a un estiloe de vida saludable y MAPA cada 1 a 2 años en pacientes con diagnóstico de HTA del delantal blanco ${ }^{12}$.

\section{HTA enmascarada}

Es aquella en que los pacientes presentan PA clínicas $<$ p95 (o $<130 / 80$ en niños $\geq 13$ años), pero PA en rango de HTA y sobrecarga $>25 \%$ en el MAPA, ya sea en el período diurno y/o nocturno. En pediatría, en niños con factores de riesgo de HTA, ésta tiene una prevalencia alrededor de un $10 \%{ }^{16}$. Cabe destacar que esta condición es diagnosticada exclusivamente mediante MAPA. Este término también puede ser aplicado a pacientes con tratamiento antihipertensivo y que presentan HTA no controlada enmascarada al MAPA ${ }^{17}$. La HTA enmascarada es considerada un fenotipo de alto riesgo cardiovascular y de daño de órgano blanco. Los hábitos de vida saludable tendrían un impacto en la disminución de su prevalencia. Así mismo los niños obesos presentan una mayor prevalencia de este tipo de $\mathrm{HTA}^{17,18}$. En adultos la tendencia actual es iniciar tratamiento antihipertensivo dado que la HTA enmascarada sería un importante factor de riesgo cardiovascular $^{19,20,21}$. En niños sugerimos iniciar tratamiento en caso de daño de órgano blanco y/o pacientes con alto riesgo cardiovascular como: ERC, DM, prematurez extrema y antecedentes familiares de eventos cardiovasculares a temprana a edad. En pacientes con HTA enmascarada sin factores de riesgo y sin daño de órgano blanco se sugiere hábitos de vida saludable y realizar seguimiento con MAPA a los 6-12 meses.

\section{Obesidad y MAPA}

Dado el gran aumento que se ha producido en la prevalencia de obesidad a nivel mundial y especialmente en nuestro país en los últimos años, nos parece importante incluir este tema y describir los hallazgos más frecuentes en MAPA

Dentro de lo más frecuente está la ausencia de dip nocturno descrito en un 30-40\% de niños obesos ${ }^{22,23}$. En edad adulta la ausencia de dip se asocia a mal pronóstico cardiovascular, renal y diabético. Cabe destacar que esta ausencia de dip también ha sido descrita en niños con sobrepeso ${ }^{24}$.

Un estudio en adolescentes que compara obesos con y sin hipertensión, y niños con peso normal, se encontró una asociación entre obesidad y mayor promedio de 24 horas de PA sistólica y diastólica y mayor sobrecarga nocturna sistólica y diastólica ${ }^{25}$. Está descri- to que hasta un $50 \%$ de los MAPA realizados a obesos tienen alguna alteración (PA elevada, hipertensión, ausencia dip nocturno $)^{26}$.

\section{IV.HTA Nocturna}

La incorporación del monitoreo ambulatorio de presión arterial, como instrumento de registro de PA, ha permitido conocer mejor los patrones de PA durante la noche, detectando ausencia de dip nocturno, HTA nocturna asociada a HTA diurna e HTA nocturna aislada. De esta forma, diversos estudios realizados en adultos, han detectado que la PA nocturna tendría relevancia clínica. Hassen describió que HTA nocturna tiene un valor pronóstico superior a la PA diurna o PA promedio de 24 horas $^{27}$. Es decir, es un predictor de complicaciones asociadas a variaciones de PA. Estas alteraciones podrían estar asociadas a trastornos en la modulación del sistema simpático, a alteraciones de los baroreceptores, a apnea del sueño y a sensibilidad diferente a la $\mathrm{sal}^{27}$.

Estudios clínicos en pacientes con ERC, la HTA nocturna y la ausencia de dip nocturno, se asocian a deterioro de la filtración glomerular y a proteinuria. En pediatría existen estudios en pacientes diabéticos en los cuales se ha demostrado que la presencia de HTA nocturna se asocia a microalbuminuria y a elevación del índice del grosor intima-media carotídeo. Cabe destacar que la HTA nocturna es un patrón frecuente de encontrar en MAPA de pacientes pediátricos diabéticos, trasplantados y con síndrome de apnea obstructiva del sueño ${ }^{28-30}$. Otro patrón detectado en MAPA es la HTA nocturna aislada (HNA), la cual se caracteriza por PA normal durante el día y PA elevada durante la noche. La HNA se ha asociado a mayor riesgo cardiovascular y daño de órgano blanco en relación a HTA diurna ais$\operatorname{lada}^{28}$. La prevalencia de HNA sería aproximadamente de $16 \%$, en niños y en adultos entre un 3 a $23 \%{ }^{28}$.

Finalmente, es importante considerar que se requieren mayores estudios para determinar la prevalencia de HTA nocturna e HNA, y el valor pronóstico de estas entidades en distintos grupos etarios y en diferentes etnias.

\section{Dip nocturno ausente}

Es un patrón dentro de MAPA que se ha asociado con la presencia de obesidad, síndrome de apnea osbtructiva del sueño y resistencia a la insulina. En población adulta se relaciona a peor evolución cardiovascu$\operatorname{lar}^{18}$. En pediatría existen estudios que no han logrado demostrar su asociación con HVI ${ }^{22,31}$.

\section{Sobrecarga aislada}

El reporte de sobrecarga de PA corresponde al porcentaje de cifras tensionales que exceden los puntos de corte prefijados como normales. En adultos, cargas sobre $40 \%$ son consideradas como anormales, aunque 
algunas publicaciones consideran desde 25\% como carga elevada. El significado de una carga alta es aún incierto. En adultos, no se ha demostrado que la sobrecarga aporte mayor predictibilidad de riesgo que las cifras de PA por sí solas. Por lo tanto la recomendación, en adultos, considera exclusivamente los valores de promedio de presiones para diagnosticar $\mathrm{HTA}^{21}$.

Mientras que, en población pediátrica, la sobrecarga de PA $(\geq 25 \%)$, además de estratificar la hipertensión como severa al encontrarse por sobre $50 \%$, permite clasificar al paciente en un estado de pre-hipertensión, cuando los promedios de PA están bajo el p95, es decir "no logra" ser clasificado como hipertenso. Sin embargo, esta clasificación deja un grupo importante de pacientes que no calzan en ningún diagnóstico preciso, de acuerdo a las recomendaciones expuestas en la tabla $\mathrm{N}^{\circ} 3$. Estos pacientes deben ser evaluados caso a caso y clasificados de acuerdo con sus factores de riesgo cardiovascular individuales $^{18}$.

\section{Hipotensión en MAPA}

No existen recomendaciones específicas respecto al límite inferior de presión arterial en MAPA. Las publicaciones existentes hacen referencia a valores de corte de presiones de reposo, basadas en las tablas previas del año $2004^{32}$. Algunos artículos definen hipotensión absoluta de acuerdo a criterios de shock en paciente crítico, basado en fórmulas de PALS o equivalentes ${ }^{33}$. La guía de recomendaciones para adultos propone cifras de corte en pacientes que han sufrido accidentes coronarios agudos, bajo las cuales la evolución posterior empeora; sin embargo, no parecen ser representativas de nuestra población ${ }^{34}$. Por lo tanto, no podemos definir cifras claras para diagnosticar hipotensión en MAPA en pacientes pediátricos. En caso necesario, se sugiere al menos informar si los promedios se encuentran sobre o bajo el percentil 50 de referencia publicado en las tablas recomendadas.

\section{Pacientes no categorizados en clasificación diagnóstica}

Aún la literatura no es concluyente en este tipo de pacientes por lo que se sugiere interpretar el MAPA caso a caso. Se debe considerar la presencia de causas secundarias de HTA, como es el caso de la ERC u otros factores de riesgo cardiovascular específicos, para clasificarlos en alguna categoría determinada por los riesgos que conlleva para la presencia de daño de órgano blanco, específicamente $\mathrm{HVI}^{7}$.

\section{Recomendaciones para el seguimiento con MAPA}

Es importante mencionar que MAPA es útil en el seguimiento de patologías que presentan mayor riesgo de HTA, por lo que se recomienda repetir MAPA en las siguientes situaciones:

1) Evaluar la eficacia de la terapia antihipertensiva

a. Confirmar el control de PA en pacientes con tratamiento médico, especialmente en aquellos con HTA secundaria. Se recomienda realizar en forma anual.

b. Evaluar aparente resistencia a los medicamentos.

c. Evaluar síntomas sugerentes de hipotensión $2^{\text {a }}$ al tratamiento farmacológico ${ }^{7}$.

2) Uso rutinario de MAPA en niños y adolescentes con patología de alto riesgo cardiovascular, tales como enfermedad renal crónica, diabetes mellitus 1 y 2, trasplante de órgano sólido, obesidad, SAOS, Coartación de aorta, síndromes genéticos asociados con HTA, tales como neurofibromatosis, síndrome de Turner o Síndrome de Williams, o en $\mathrm{RNPT}^{12}$.

a. En pacientes con ERC, diálisis, trasplante de órgano sólido y coartación de la aorta se recomienda realizar MAPA anual ${ }^{12}$.

b. En pacientes con diabetes mellitus se recomienda realizar cada 6 a 12 meses, en busca de hipertensión nocturna aislada ${ }^{35}$.

3) En HTA de delantal blanco, se recomienda repetir MAPA en 1 a 2 años ${ }^{12}$.

4) En HTA enmascarada, se recomienda repetir $\mathrm{MAPA}^{36}$. En nuestra opinión, sería prudente repetirlo a los 6-12 meses.

5) En aquellos pacientes que han sido sometidos a MAPA y su resultado es "borderline" para el diagnóstico de HTA, se sugiere repetir examen ${ }^{36}$.

\section{Conclusión}

El diagnóstico y tratamiento oportuno de la HTA en la población pediátrica es importante en la prevención de futuras enfermedades cardiovasculares y muerte en la adultez temprana, lo que tendría impacto en la salud pública.

El MAPA es una herramienta útil en el diagnóstico y caracterización de la HTA, en especial en aquellos pacientes que tienen factores de riesgo para daño de órgano blanco, en los cuales este examen debería ser un pilar en el seguimiento habitual.

Existen recomendaciones internacionales en la población pediátrica, que debieran actualizarse y resolver, entre otras cosas, el manejo de aquellos pacientes no categorizados según la clasificación diagnóstica de MAPA. Asimismo, sería ideal contar con nuevas tablas de referencias con población local, dado que las tablas usadas actualmente fueron realizadas en población europea. Sería importante también desarrollar evidencia 
en cuanto a la relación existente entre alteraciones en MAPA y resultados clínicos a largo plazo.

Por este motivo es fundamental unificar criterios locales para la interpretación de este examen y realizar estudios de investigación colaborativos que puedan aportar mayor información acerca de MAPA en pediatría.

\section{Conflicto de intereses}

Los autores declaran no tener conflicto de intereses

\section{Referencias}

1. Siu AL. Screening for high blood pressure in adults: U.S. Preventive Services Task Force recommendation statement. Ann Intern Med. 2015;163(10):778-86.

2. Salice P, Ardissino G, Barbier P, et al. Differences between office and ambulatory blood pressures in children and adolescents attending a hospital hypertension clinic. J Hypertens. 2013;31(11):2165-75.

3. Stergiou GS, Alamara CV, Salgami EV, Vaindirlis IN, Dacou-Voutetakis C, Mountokalakis TD. Reproducibility of home and ambulatory blood pressure in children and adolescents. Blood Press Monit. 2005;10(3):143-7.

4. Seeman T, Palyzova D, Dusek J, Janda J. Reduced nocturnal blood pressure dip and sustained nighttime hypertension are specific markers of secondary hypertension. J Pediatr. 2005;147(3):36671.

5. Bjelakovic B, Jaddoe VW, Vukomanovic $\mathrm{V}$, et al. The relationship between currently recommended ambulatory systolic blood pressure measures and left ventricular mass index in pediatric hypertension. Curr Hypertens Rep. 2015;17(4):534.

6. Conkar S, Yilmaz E, Hacikara S, Bozabali S, Mir S. Is Daytime Systolic Load an Important Risk Factor for Target Organ Damage in Pediatric Hypertension? J Clin Hypertens (Greenwich, Conn). 2015;17(10):760-6.

7. Flynn JT, Daniels SR, Hayman LL, et al. Update: ambulatory blood pressure monitoring in children and adolescents: a scientific statement from the American Heart Association. Hypertension (Dallas, Tex: 1979). 2014;63(5):1116-35.

8. Samuels J, Ng D, Flynn JT, et al. Ambulatory blood pressure patterns in children with chronic kidney disease. Hypertension (Dallas, Tex : 1979). 2012;60(1):43-50.

9. Mitsnefes M, Flynn J, Cohn S, et al. Masked hypertension associates with left ventricular hypertrophy in children with CKD. J Am Soc Nephrol: JASN. 2010;21(1):137-44.

10. Gupta D, Chaturvedi S, Chandy S, Agarwal I. Role of 24-h ambulatory blood pressure monitoring in children with chronic kidney disease. Indian J Nephrol. 2015;25(6):355-61.

11. Urbina E, Alpert B, Flynn J, et al. Ambulatory blood pressure monitoring in children and adolescents: recommendations for standard assessment: a scientific statement from the American Heart Association Atherosclerosis, Hypertension, and Obesity in Youth Committee of the council on cardiovascular disease in the young and the council for high blood pressure research. Hypertension (Dallas, Tex : 1979). 2008;52(3):433-51.

12. Flynn JT, Kaelber DC, Baker-Smith CM, et al. Clinical Practice Guideline for Screening and Management of High Blood Pressure in Children and Adolescents. Pediatrics. 2017;140(3).

13. Jurko A, Jr., Jurko T, Minarik M, et al. Endothelial function in children with white-coat hypertension. Heart Vessels. 2018;33(6):657-63.

14. Tokgoz ST, Yilmaz D, Tokgoz Y, Celik $B$, Bulut $Y$. The evaluation of arterial stiffness of essential hypertension and white coat hypertension in children: a case-control study. Cardiol Young. 2018;28(3):403-8.

15. Miyashita Y, Flynn JT, Hanevold CD. Diagnosis and management of white-coat hypertension in children and adolescents: A Midwest Pediatric Nephrology Consortium study. J Clinl Hypertens (Greenwich, Conn). 2017;19(9):884-9.

16. Iturzaeta A, Pompozzi L, Casas Rey C, Passarelli I, Torres F. Prevalence of masked hypertension among children with risk factors for arterial hypertension. Arch Argent Pediatr. 2018;116(5):328-32.

17. Anstey DE, Pugliese D, Abdalla M, Bello NA, Givens R, Shimbo D. An Update on Masked Hypertension. Curr Hypertens Rep. 2017;19(12):94.

18. Macumber I. Ambulatory Blood Pressure Monitoring in Children and Adolescents: a Review of Recent Literature and New Guidelines. Curr Hypertens Rep. 2017;19(12):96.

19. Banegas JR, Ruilope LM, de la Sierra A, et al. Relationship between Clinic and Ambulatory Blood-Pressure Measurements and Mortality. N Engl J Med. 2018;378(16):1509-20.

20. Franklin SS, O'Brien E, Staessen JA.
Masked hypertension: understanding its complexity. Eur Heart J. 2017;38(15):1112-8.

21. Prat H AA, Alarcón G, et al Guías para la monitorización ambulatoria de presión arterial de 24 horas. Documento de la Sociedad Chilena de Cardiología y Cirugía Cardiovascular. Rev Chil Cardiol 2017;36:264-74

22. Macumber IR, Weiss NS, Halbach SM, Hanevold CD, Flynn JT. The Association of Pediatric Obesity With Nocturnal NonDipping on 24-Hour Ambulatory Blood Pressure Monitoring. Am J Hypertens. 2016;29(5):647-52.

23. Westerstahl M, Hedvall Kallerman P, Hagman E, Ek AE, Rossner SM, Marcus C. Nocturnal blood pressure non-dipping is prevalent in severely obese, prepubertal and early pubertal children. Acta Paediatr (Oslo, Norway : 1992). 2014;103(2):22530.

24. Giacchi V, Timpanaro T, Lo Presti D, et al. Prehypertension in adolescents with cardiovascular risk: a comparison between type 1 diabetic patients and overweight subjects. BMC Res Notes. 2016;9:122.

25. Dibeklioglu SE, Cevik BS, Acar B, et al. The association between obesity, hypertension and left ventricular mass in adolescents. J Pediatr Endocrinol Metab: JPEM. 2017;30(2):167-74

26. Strojny W, Drozdz D, Fijorek K, et al. Looking for new diagnostic tools and biomarkers of hypertension in obese pediatric patients. Blood Press Monit. 2017;22(3):122-30.

27. Hansen TW, Li Y, Boggia J, Thijs L, Richart T, Staessen JA. Predictive role of the nighttime blood pressure. Hypertension (Dallas, Tex : 1979). 2011;57(1):3-10.

28. Fujita H, Matsuoka S, Awazu M. Masked Isolated Nocturnal Hypertension in Children and Young Adults. Pediatr Cardiol. 2018;39(1):66-70.

29. Lee SH, Kim JH, Kang MJ, Lee YA, Won Yang S, Shin CH. Implications of nocturnal hypertension in children and adolescents with type 1 diabetes. Diabetes Care. 2011;34(10):2180-5.

30. McGlothan KR, Wyatt RJ, Ault BH, et al. Predominance of nocturnal hypertension in pediatric renal allograft recipients. Pediatr Transplant.. 2006;10(5):558-64. 
31. Seeman T, Hradsky O, Gilik J. Nocturnal blood pressure non-dipping is not associated with increased left ventricular mass index in hypertensive children without end-stage renal failure. Eur J Pediatr. 2016;175(8):1091-7.

32. Banker A, Bell C, Gupta-Malhotra M, Samuels J. Blood pressure percentile charts to identify high or low blood pressure in children. BMC Pediatr. 2016;16:98.
33. Haque IU, Zaritsky AL. Analysis of the evidence for the lower limit of systolic and mean arterial pressure in children. Pediatr Crit Care Med. 2007;8(2):138-44.

34. Divison-Garrote JA, Banegas JR, De la Cruz JJ, et al. Hypotension based on office and ambulatory monitoring blood pressure. Prevalence and clinical profile among a cohort of 70,997 treated hypertensives. J Am Soc Hypertens. 2016;10(9):714-23.
35. Lurbe E, Agabiti-Rosei E, Cruickshank JK, et al. 2016 European Society of Hypertension guidelines for the management of high blood pressure in children and adolescents. J Hypertens. 2016;34(10):1887-920.

36. O’Brien E, Parati G, Stergiou G, et al. European Society of Hypertension position paper on ambulatory blood pressure monitoring. J Hypertens. 2013;31(9):1731-68. 